\title{
Day case superficial parotidectomy—does it work?
}

\author{
Mark Edmond ${ }^{1,2} \cdot$ Elaine Campbell ${ }^{2} \cdot$ Venkat Reddy $^{2}$
}

Received: 28 November 2020 / Accepted: 21 January 2021 / Published online: 8 February 2021

(c) The Author(s), under exclusive licence to Springer-Verlag GmbH, DE part of Springer Nature 2021

\begin{abstract}
Purpose To establish if day case superficial parotidectomy is feasible, safe and does not result in excess readmissions. Method A retrospective review was carried out of all patients listed for superficial parotidectomy with day case intent by a single surgeon between January 2016 and December 2019 inclusively. The reasons for failure of same day discharge were established. Postoperative complications and readmissions were recorded. Our approach for a superficial parotidectomy typically includes the use of a $10 \mathrm{Fr}$ suction drain which is removed at $4 \mathrm{~h}$ postoperatively if the output is less than $30 \mathrm{ml}$.

Results Ninety-one consecutive superficial parotidectomies listed for day case surgery were eligible for inclusion. Seventeen patients failed to be discharged on the same day and were admitted giving a day case success rate of $81 \%$. Most of these $(n=9)$ occurred in the first year of adopting day case surgery. The most common reason to admit patients was a late finish $(n=8,47 \%)$. Six patients (25\%) were admitted due to anaesthetic complications. One patient had a surgical complication requiring admission.

Conclusion Our series demonstrates that day case superficial parotidectomy using a surgical drain is feasible, safe and does not result in an unacceptable readmission rate. In our experience, surgical complications are an uncommon cause for day case failure. The most common cause for day case failure was a late finish. Postoperative complications including bleeding, seroma/salivary collection and facial nerve palsy were in keeping with or better than those quoted in the literature.
\end{abstract}

Keywords Parotidectomy $\cdot$ Outpatient $\cdot$ Day case surgery $\cdot$ Salivary gland $\cdot$ Outcomes

\section{Introduction}

It is the practice of most Head and Neck units in the UK to perform superficial parotidectomy as an inpatient procedure, generally with the use of a surgical drain and an inpatient stay of 1-2 days [1]. There is a longstanding trend for surgeons to increase the number of procedures performed as day case or outpatient, whilst maintaining the highest standards of patient safety and good outcomes [2]. The operational difficulties and service pressures faced by

Mark Edmond

mark.edmond@nhs.net

Elaine Campbell

elaine.campbel113@nhs.net

Venkat Reddy

venkat.reddy1@nhs.net

1 Poole Hospitals NHS Foundation Trust, Longfleet Road, Poole, Dorset BH15 2JB, UK

2 ENT Department, Royal Cornwall Hospitals NHS Trust, Treliske, Truro TR1 3LJ, Cornwall, UK the NHS in recent times are well documented. In the Winter of 2017-2018, NHS England recommended that all elective surgery be cancelled for the month of January to free up bed capacity for emergency admissions [3]. These pressures have been amplified by the worldwide Coronavirus pandemic which has reduced inpatient capacity and led to thousands of cancelled procedures. NHS waiting times are at record highs [4]. Superficial parotidectomies are especially at risk of cancellation on the day of surgery as they are generally listed outside of cancer pathways; approximately $80 \%$ of all parotid lumps are benign [5]. In our unit in 2015, 1 in 5 superficial parotidectomies were cancelled on the day of surgery due to bed shortages.

On-the-day cancellations negatively impact on patient experience, outcomes and hospital flow [6]. When coupled with diagnostic uncertainty, the risk of malignant transformation over time, and patient preference, there is a clear need to evaluate the feasibility of day case superficial parotidectomy in the UK. We present 4 years of experience of performing day case superficial parotidectomies in a UK district general hospital. 


\section{Background}

Day case superficial parotidectomy has been described in the literature for over 25 years with Steckler et al. (Texas, USA) publishing their series of 56 outpatient parotidectomies in 1991 [7]. However, there is a paucity of data from the UK and this is reflected in a recent meta-analysis comparing day case vs inpatient parotidectomy in which no UK studies were deemed appropriate for inclusion [8]. We present the experience of a Head and Neck surgeon in the South West of England, whose practice is to perform superficial parotidectomy as a day case procedure in patients preoperatively assessed as appropriate for day case surgery.

\section{Objectives}

1. What was the rate of same day discharges?

2. What were the causes for failure of same day discharge?

3. What were the complications?

4. What was the readmission rate?

\section{Materials and methods}

Following registration and approval by our local Clinical Effectiveness and Quality Improvement department, we performed a retrospective service evaluation by reviewing the electronic patient record (EPR) of all patients who underwent a superficial parotidectomy under the care of the senior author between January 2016 and December 2019 inclusively. January 2016 represents the date from which the senior author changed practice to performing day case superficial parotidectomy. The study was stopped prior to the period of significant disruption to elective surgery caused by the Coronavirus pandemic. Patients were identified from handwritten theatre logbooks, and correlated with electronic theatre logbooks and handwritten theatre booking diaries. This identified 121 patients. Patients were excluded if they were planned as an inpatient for surgical reasons (i.e. if they were undergoing a neck dissection, lymph node sampling, or radical parotidectomy). Patients who were pre-assessed as not appropriate for day case surgery (due to comorbidities or social circumstances) were excluded. This left a total of 91 patients eligible for inclusion (Fig. 1).

The time stamped "care episode" section of the EPR was subsequently interrogated to ensure the date of discharge and the date of admission were the same. This was corroborated by reviewing the electronic discharge summary. If the patient had not been successfully discharged the same day, their physical case notes including operative record, anaesthetic record, clinical notes and nursing

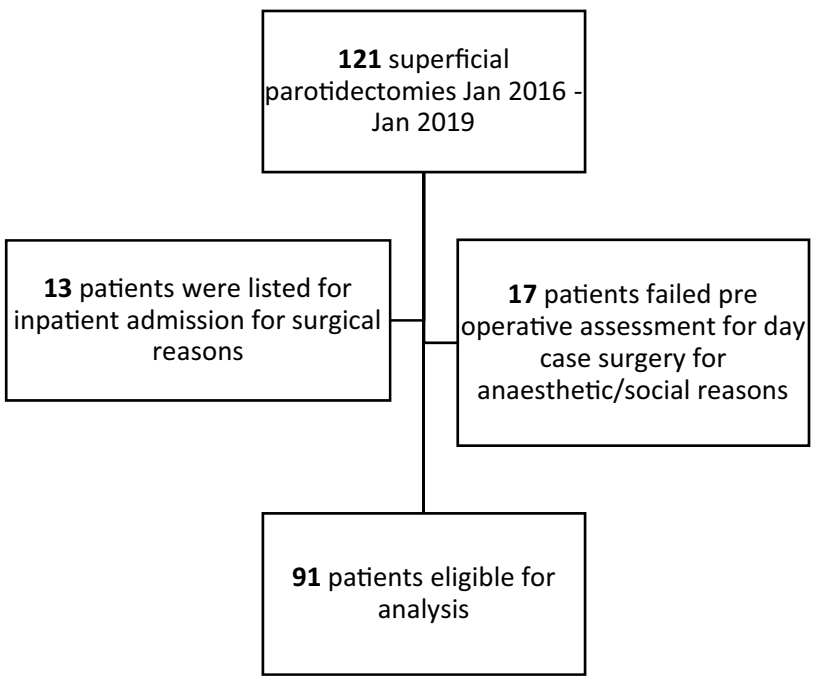

Fig. 1 Flow chart to demonstrate patient selection

notes were then analysed to identify the cause for day case failure. All patients had their EPR scrutinised for the following: Emergency Department attendances, GP attendances, ENT treatment room attendances and if available, their follow-up ENT clinic letter. Documentation from the above was used to identify postoperative complications and re-admission to hospital. Strengthening The Reporting of Observational Studies in Epidemiology (STROBE) reporting guidelines were followed [9].

Superficial partial parotidectomy is the method typically employed by our department. A modified Blair incision is planned with a skin marker and local anaesthetic infiltration is performed with $10 \mathrm{ml}$ of $0.5 \%$ bupivacaine with 1 in 200,000 adrenaline $(25 \mathrm{mg}$ of bupivacaine and $0.05 \mathrm{mg}$ of adrenaline). The Inomed C2 ${ }^{\mathrm{TM}}$ (Inomed Medzintechnik $\mathrm{GmbH}$, Emmendingen, Germany) four-channel facial nerve monitor is employed. The skin flap is raised and the trunk of the facial nerve is identified. The nerve branches are typically followed in an antegrade fashion to excise the lesion with a cuff of normal parotid where possible. Harmonic Scalpel ${ }^{\mathrm{TM}}$ (Ethicon Inc., Somerville, New Jersey) is used for mobilising and dissecting the parotid. A 10Fr Blakes suction drain is placed underneath the superficial musculoaponeurotic (SMAS) layer. Closure is performed with 3-0 Vicryl Rapide ${ }^{\mathrm{TM}}$ (Ethicon Inc., Somerville, New Jersey) to the SMAS layer and 4-0 Monocryl ${ }^{\mathrm{TM}}$ (Ethicon Inc., Somerville, New Jersey) to skin. No dressings are used, and chloramphenicol ointment is applied to the wound twice a day until the tube runs out. The drain is removed at 4-h post-op if the output is less than $30 \mathrm{ml}$. Patients are discharged if they meet the Hospital's standard day case discharge criteria (Appendix A) and following review by the surgical team. All patients receive a detailed discharge summary with information (Appendix 
Table 1 Causes for failure of same day discharge

\begin{tabular}{ll}
\hline Cause for day case failure & Number of patients (total=17) \\
\hline Late finish $^{\mathrm{a}}$ & $8(47 \%)$ \\
Anaesthetic & $6(35 \%)$ \\
& Two developed urinary retention requiring catheterization \\
& Four with cardiorespiratory instability intraoperatively or in recovery \\
Inappropriate for day case & \\
& $2(12 \%)$ \\
Surgical & Two patients with inadequate home support \\
& $1(6 \%)$ \\
& One patient with a CN VII palsy and pharmacy unable to provide \\
& appropriate eye drops, therefore admitted \\
\hline
\end{tabular}

a Defined as finishing after 17:30 and no other obvious cause documented for admission

${ }^{\mathrm{b}}$ Social issues not picked up at pre-assessment e.g. patient did not have a competent adult at home with them for $24 \mathrm{~h}$ following their operation

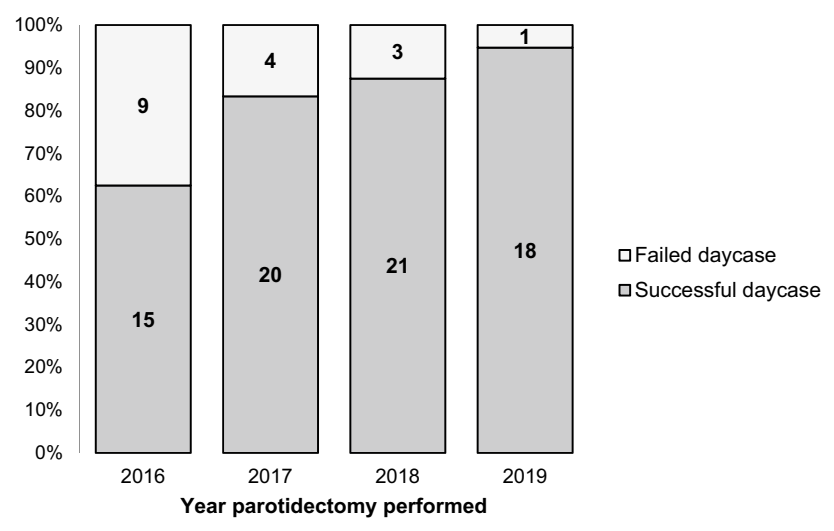

Fig. 2 Day case success rate by year 2016-2019 inclusive

B) regarding their postoperative recovery and actions to take in the event of complications.

\section{Results}

Ninety-one consecutive superficial parotidectomies listed for day case surgery were eligible for inclusion. Seventeen patients failed to be discharged on the same day and were admitted. Table 1 documents the causes identified for day case failure in these 17 patients.

An overall day case success rate of $81 \%$ was observed. Progressive yearly improvement was also noted (Fig. 2), ranging from $63 \%$ in year one to $95 \%$ in year four of the analysis period.

Of the 17 patients who failed to be managed as day cases, 2 patients were readmitted within 30 days of their procedure. Both were for postoperative wound infections requiring IV antibiotics and in one case, incision and drainage of an abscess. One failed same day discharge due to a late finish and the other because of anaesthetic complications. There were no readmissions from the group of patients who were
Table 2 Surgical complications for all patients

\begin{tabular}{ll}
\hline Complication & $\begin{array}{l}\text { Number } \\
\text { of patients } \\
(n=27)^{\mathrm{a}}\end{array}$ \\
\hline Postoperative wound infections managed by GPs & $8(9 \%)$ \\
Postoperative wound infections managed by ENT & $3(3 \%)$ \\
Transient facial nerve palsy resolved at follow-up & $9(10 \%)$ \\
Facial nerve palsy not resolved at time of follow-up & $1(1 \%)$ \\
Seroma/sialocele/salivary fistula $^{\mathrm{b}}$ & $3(3 \%)$ \\
Frey's Syndrome $_{\text {Chronic pain }}$ & $2(2 \%)$ \\
\hline
\end{tabular}

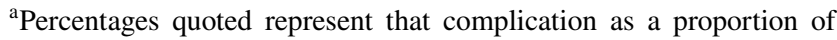
the whole cohort (91 patients)

${ }^{\mathrm{b}}$ The vast majority of authors do not differentiate between these complications as they are generally managed in the same manner[7]

successfully managed as day cases. Surgical complications for all patients are summarised in Table 2. We have subclassified wound infection in those diagnosed and managed by GPs and those that required ENT input.

\section{Discussion}

We report an overall day case success rate of $81 \%$ with year on year improvement (Fig. 2.), ranging from $63 \%$ in the first year to $95 \%$ in the fourth year since day case parotidectomy was introduced. Success rates quoted in the literature range from $47-95.8 \%[10,11]$. Large data sets for other procedures converted to day case in recent times report similar rates; thyroid lobectomy ( $80 \%)$, laparoscopic cholecystectomy (75.8\%) and laparoscopic fundoplication (81.6\%) [12-14]. The majority (53\%) of our day case failures occurred within a year of changing practice to a day case model. As with all changes in practice, there is often a time lag before optimal performance is achieved [15]. 
The most common cause that we identified for day case failure was a late finish. Our day case surgery and recovery unit closes at 22:00. As a consequence, any case which finished after 17:30 with our standard postoperative instructions would require their drain to be assessed for removal after the day case unit closed. In this scenario, the day case staff arrange for the patient to be admitted to an inpatient ward. It is difficult to interpret the exact sequence of events from retrospective analysis of patients' notes. However, anecdotally, it would seem that the impetus for same day discharge rapidly diminishes once a patient reaches an inpatient bed. This is likely due to the work load of nursing staff and the social norm to avoid discharging patients late in the evening.

In our experience, excessive drain output or excessive bleeding in the 4-h period following surgery is not a common occurrence. No patients required admission for excessive drain output. This would raise the question as to whether our patients experienced problems later in their postoperative course due to haematoma or seroma/salivary collection/ fistula or infection. We report a collection rate of $3 \%$ which is in keeping with the rates quoted in the literature, as shown in Table 3. As with many authors, it is not our practice to send any non-purulent aspirate fluid from collections for amylase, hence "collection" encompasses sialocele/salivary fistula as well as seroma [8]. Our cumulative postoperative wound infection rate is higher $(12 \%)$ than other data sets. However, as highlighted in Table 2, the majority of these ( 8 out of 11) were diagnosed and managed in primary care by GPs. This high incidence of GP diagnosed infections may explain our slightly higher rate, given that mild erythema and swelling caused by normal wound healing can be misdiagnosed as infection. Equally, seroma/salivary collection/ salivary fistula can present in similar ways to wound infection. Consequently, it may be that our true postoperative collection rate is slightly higher, and our surgical site infection rate slightly lower than quoted. The three remaining wound infections required ENT input, with two requiring re-admission as discussed later.

Despite our low rate of postoperative collection, some may argue that the time interval of $4 \mathrm{~h}$ between operation and removal of drain (if the output is less than $30 \mathrm{ml}$ ) is too short. There is little definitive evidence regarding drain removal timing, and practice varies widely. Mofle et al. perhaps provide the best evidence and report a median total drainage of $35 \mathrm{ml}$ in their series of 96 superficial parotidectomies [16]. Equally, some authors advocate a drainless parotidectomy as a method to promote day case success, generally using tissue sealant or topical haemostatic agent [1]. Our feeling is that if bleeding is going to occur, it is likely to occur within the first $4 \mathrm{~h}$ following surgery. We see the function of the suction drain as twofold; to alert us to bleeding and to encourage the skin flap to adhere to the deeper tissues.

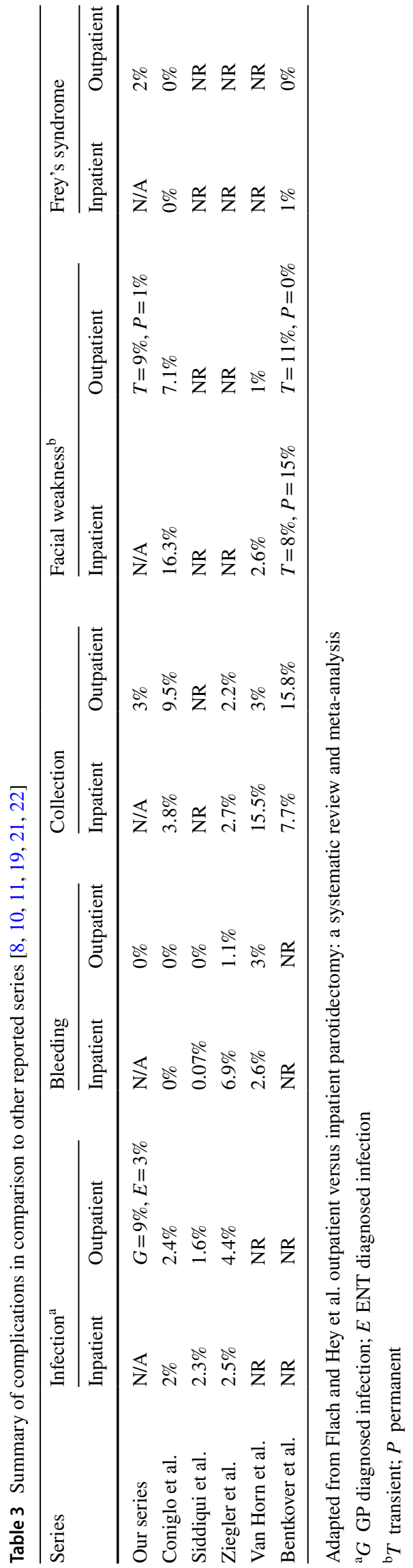


Other complications such as facial nerve weakness, both temporary and permanent, is in line with the literature [17, 18]. Frey's Syndrome is an uncommon complication and we report two cases. Two patients (2\%) were readmitted for surgical site infections. Both required IV antibiotics, with one patient requiring incision and drainage of an abscess under local anaesthetic. Both patients had comorbidities predisposing them to wound infections (type 2 diabetes and obesity) and were from the group that failed same day discharge. Siddiqui et al. quote a readmission rate of $1.3 \%$ [19]. For comparison with other Head and Neck day case procedures, the readmission rates quoted from studies used in the American Thyroid Association statement supporting outpatient thyroidectomy ranges $0-3.9 \%$ [20].

A health economics analysis is beyond the scope of this study; however, the UK National tariff payment system estimates the cost of an inpatient bed on a surgical ward in 2018-2019 to be $£ 241 /$ night [23]. Over the course of 4 years, this would represent a total saving of $£ 17,834$ based on bed costs alone. This is before even considering more efficient theatre utilisation and flow of admissions through the wider hospital. If we extrapolate our 2015 cancellation rate of $20 \%$ for inpatient parotidectomy, we have prevented 18 patients from having their operation cancelled on the day of surgery. Patient centred benefits for day case surgery include allowing recovery in a familiar environment, less separation from family, greater convenience for workers or patients with childcare commitments and reduced risk of hospital acquired infections [24]. All of these factors are more pertinent in the context of the worldwide Coronavirus pandemic.

\section{Conclusions}

The most significant barrier to successful day case discharges is a late finish and the subsequent difficulties in discharging patients from inpatient wards late at night. Surgical problems such as excessive bleeding or drain output were uncommon causes for day case failure. Our series would appear to have a higher rate of postoperative infections; however, this includes infections diagnosed by non-ENT clinicians. All other complications including that of postoperative collections were in keeping with, or better than those quoted in the literature. Our series demonstrates that day case superficial parotidectomy is feasible, safe and does not result in excessive re-admission and allows patients to benefit from the already established patient centred benefits of day case surgery.

\section{Appendix A}

\section{Standard hospital day case discharge criteria}

Patients are considered as fit for discharge from our day case unit if the following criteria have been met.

- Observations are taken on the arrival of the patient to the day case ward and then every hour, for $4 \mathrm{~h}$. Observations need to be within normal limits at $4 \mathrm{~h}$.

- Passed urine

- Tolerate fluids and light diet

- Wound has no significant ooze

- Drain removed as per postoperative instructions

Additionally, patients are asked to confirm that they will adhere to the following:

- There is a responsible adult who is able to care for them for $24 \mathrm{~h}$ following their operation

- Will not drive or ride a bike or motorcycle

- Will not operate machinery or appliances such as cookers

- Will not drink alcohol

- Will not lock the bathroom or lavatory door in case they need assistance

- Will drink plenty of fluids and eat a light diet

- Will not make important decisions, sign a cheque or other documents

\section{Appendix B}

Soluble sutures used so they do not need to be removed. If facial weakness develops following surgery, it usually settles over a few months, but can take up to 12 months. If the weakness persists beyond that, it may be permanent. Numbness around the side of the head is normal, it usually decreases in size to affect only the lower ear over a couple of months. Rarely, the wound may break down and open up-please contact the ward so that we can arrange to see you in this situation. Rarely, there may be significant bleeding after surgery. If you develop a progressively enlarging swelling that is bigger than a golf ball, please contact the ward so we can arrange to see you. The wound will usually swell in the first few weeks as it heals.

Apply choloramphenicol ointment twice a day with a clean finger until the tube runs out. You should otherwise keep the wound dry for the first 4 days, then it should be fine to wash gently with soap and warm water. After 7 days, use BioOil or E45 (or equivalent) to massage the wound to reduce the swelling. 
Some patients will have complications following surgery such as wound infection, where the wound may become red and pus may exude - please see your GP to assess and manage this in the first instance-they will contact us if they feel we need to take a look at it.

Some may experience a leaky wound - this usually dries up over a few months, a dressing can be applied to soak up the discharge until it stops. Some may experience a collection of fluid under the skin which will need to be drawn off with a needle, this usually recurs until stopping over the course of a few months. Please contact the ward so that we can arrange to see you in this situation.

I will contact you with the results of the histology when available and arrange any follow-up required thereafter. This may just be in the form of a phone conversation.

Ward contact details:

Funding No funding was received for this project.

Data availability The authors confirm that the data supporting the findings of this study are available within the article and its supplementary materials.

\section{Compliance with ethical standards}

Conflicts of interest All authors have no conflict of interest to declare.

Ethical considerations This project was approved by our local audit and quality improvement department.

\section{References}

1. Cunniffe HA, Wong BLK, Hilger AW, Burgan OT (2019) Drainfree parotidectomy: a pilot study using ARTISS fibrin sealant. Eur Arch Otorhinolaryngol 276(7):2025-2029

2. Audit commission for local authorities and the National Health Service in England in Wales (1990) A short cut to better services, day surgery in England and Wales. The Audit Commission, London

3. Lay K, Smyth C, Maguire P (2018) NHS chiefs defend cancellation of thousands of operations. The Times, 3 January 2018

4. Shaun G (2020) Covid-19: waiting times in England reach record highs. BMJ 370:3557

5. Mallon DH, Kostalas M, MacPherson FJ, Parmar A, Drysdale A, Chisholm E (2013) The diagnostic value of fine needle aspiration in parotid lumps. Ann R Coll Surg Engl 95:258-262

6. Wong DJN, Harris SK, Moonesinghe SR, SNAP-2 EPICCS Collaborators (2018) Cancelled operations: a 7 day cohort study of planned adult inpatient surgery in 245 UK National Health Service Hospitals. Br J Anaesth 121(4):730-738

7. Steckler R (1991) Outpatient parotidectomy. Am J Surg 162(4):303-305

8. Flach S, Hey SY, Lim A, Maniam P, Li Z, Donnan P, Manickavasagam J (2020) Outpatient (same-day discharge) versus inpatient parotidectomy: a systematic review and meta-analysis. Clin Otolaryngol 45:529-537

9. Von Elm E, Altman DG, Egger M, Pocock SJ, Gøtzsche PC, Vandenbroucke JP (2007) The strengthening the reporting of observational studies in epidemiology (STROBE) statement: guidelines for reporting observational studies. Ann Intern Med 147:573

10. Ziegler A, Lazzara G, Thorpe E (2018) Safety and efficacy of outpatient parotidectomy. J Oral Maxillofac Surg 76(11):2433-2436

11. Bentkover SH, Kozlicak BA, Girouard S (1996) Outpatient parotidectomy at the Fallon Clinic. The first 2 years. Arch Otolaryngol Head Neck Surg 122(10):1049-1053

12. Snyder KS, Hamid KS, Robertson CR, Rai S, Bossen A, Luh $\mathrm{JH}$ et al (2010) Outpatient thyroidectomy is safe and reasonable: experience with more than 1000 planned outpatient procedures. J Am Coll Surg 210(5):575-584

13. Solodkyy A, Hakeem AR, Oswald N, Di Franco F, Gergely S, Harris AM (2018) "True day case" laparoscopic cholecystectomy in a high-volume specialist unit and review of factors contributing to unexpected overnight stay. Minim Invasive Surg 2018:1260358

14. Molina JC, Misariu AM, Nicolau I, Spicer J, Mulder D, Ferri LE, Mueller CL (2018) Same day discharge for benign laparoscopic hiatal surgery: a feasibility analysis. Surg Endosc 32(2):937-944

15. Bates DW, Kuperman GJ, Wang S, Gandhi T, Kittler A, Volk L et al (2003) Ten commandments for effective clinical decision support: making the practice of evidence-based medicine a reality. J Am Med Inform Assoc 10(6):523-530

16. Mofle P, Urquhart A (2008) Superficial parotidectomy and postoperative drainage. Clin Med Res 6(2):68-71

17. Kintworth N, Zenk J, Koch M, Iro H (2010) Postoperative complications after extracapsular dissection of benign parotid lesions with particular reference to facial nerve function. Laryngoscope 120:484-490

18. Sood A, Houlton JJ, Nguyen SA, Gillespie MB (2015) Facial nerve monitoring during parotidectomy: a systematic review and meta-analysis. Otolaryngol Head Neck Surg 152(4):631-637

19. Siddiqui S, Singh R, Siddiqui E, Zhao E, Eloy J, Baredes $\mathrm{S}$ et al (2018) Outpatient versus inpatient parotidectomy: comparison of post-operative complication rates. Laryngoscope 129(3):655-661

20. Terris DJ, Snyder S, Carnneiro-Pla D, Inabnet WB, Kandil E, Orloff L et al (2013) American Thyroid Association statement on outpatient thyroidectomy. Thyroid 23(10):1193-1202

21. Coniglio A, Deal A, Hackman T (2019) Outcomes of drainless outpatient parotidectomy. Head Neck 41(7):2154-2158

22. Van Horn A, Goldman R, Charnigo R, Johnson K, Valentino J, Aouad R (2017) Outpatient versus observation/inpatient parotidectomy: patient factors and perioperative complications. Eur Arch Otorhinolarnygol 274(9):3437-3442

23. National Tariff payment system 2017/18 and 2018/19. NHS improvement (internet) https://improvement.nhs.uk/resources/ national-tariff/. Accessed 3 March 2020

24. Ear, Nose and Throat Surgery GIRFT Programme National Specialty Report 2019 (internet) https://gettingitrightfirsttime.co.uk/ wp-content/uploads/2019/10/ENT-Report-Nov19-L-FINAL.pdf. Accessed 3 March 2020

Publisher's Note Springer Nature remains neutral with regard to jurisdictional claims in published maps and institutional affiliations. 